\title{
Relato sobre o desenvolvimento e uso de objeto de aprendizagem com elementos de aprendizagem baseada em problemas no contexto de Computação para Engenharias
}

\author{
Narci Nogueira da Silva ${ }^{1}$, Marco Aurélio Graciotto Silva ${ }^{1}$ \\ ${ }^{1}$ Universidade Tecnológica Federal do Paraná \\ Departamento Acadêmico de Computação - Campo Mourão - PR - Brasil \\ Programa de Pós-Graduação em Informática - Cornélio Procópio - PR - Brasil \\ \{narci, magsilva\}@utfpr.edu.br
}

\begin{abstract}
Problem-based learning (PBL) is a successful approach for Computing education for Engineering, Nonetheless, it is not trivial to create and disseminate educational resources that support PBL activities. Learning objects $(L O)$ can address these problems, supporting development and reuse. Using a method tailored for $L O$ development with PBL elements, our goal within this paper is to report the development of an LO about computer programming, and its implementation for a Engineering program setting. After evaluating the $P B L$ activity and the $L O$, students considered both good, and provided evidences supporting the motivation and efficacy of the learning activity.
\end{abstract}

Resumo. Considerando o ensino de computação para engenharia, a aprendizagem baseada em problemas (PBL) é uma abordagem bem sucedida. No entanto, não é trivial criar e disseminar recursos educacionais que apoiem atividades de PBL. Objetos de Aprendizagem (OA) podem abordar esses problemas, apoiando o desenvolvimento e a reutilização. Considerando um método para desenvolvimento de OA que agrega elementos de $P B L$, nosso objetivo, neste trabalho, é relatar o desenvolvimento de um OA sobre programação de computadores e a utilização do OA para um grupo de alunos de Engenharia. Após a avaliação da atividade e do OA pelos alunos, eles classificaram ambas como boas e apresentaram evidências positivas quanto à motivação e a eficácia do aprendizado.

\section{Introdução}

Estudantes normalmente têm dificuldades no entendimento dos conceitos básicos em disciplinas introdutórias à computação. Isso não acontece somente nos cursos da área de Informática, mas também de outras áreas, como Engenharias [Bosse e Gerosa 2015]. A utilização de metodologias ativas de aprendizagem possui um papel importante na resolução deste cenário. Dentre essas, destacamos a Aprendizagem Baseada em Problemas (PBL). Nesta, as atividades são centradas na resolução de problemas por parte do alunos, os quais deixam de ser receptores do conhecimento, tradicionalmente passado pelo professor, e se tornam os condutores principais do próprio aprendizado. $\mathrm{O}$ professor firma-se como facilitador para que seus alunos cumpram as etapas de resolução 
do problema apresentado e construam o conhecimento baseado em sua experiência e em novos conhecimentos pesquisados ou compartilhados pelos alunos [Gil 2015].

Não obstante a relevância de PBL, depara-se com a questão de transferir e aplicar tais atividades em diferentes contextos. Recursos educacionais abertos (REA) e objetos de aprendizagem (OA) que fomentem o reúso dos recursos para suporte às atividades de ensino [Wiley 2000], preferencialmente de forma aberta [Albright 2005], são facilitadores para o processo de transferência de atividades e experiências de aprendizagem.

Considerando iniciativas para o desenvolvimento sistemático de tais artefatos, torna-se necessária a representação de aspectos e elementos de metodologias ativas. Neste âmbito, apresentamos neste artigo um relato de experiência sobre o uso de um método para desenvolvimento de OA para utilização em atividades de PBL. Nosso objetivo é descrever a aplicação do método e o uso do objeto de aprendizagem criado.

O objeto de aprendizagem foi criado para turmas iniciais de cursos de Engenharia e trata de conceitos básicos de programação de computadores. O método é baseado no LODM [Graciotto Silva et al. 2011], uma abordagem baseada em modelos para desenvolvimento de objetos de aprendizagem. Foram incorporados itens de informação específicos para PBL e adicionadas atividades para auxiliar a definição desses itens. Após o desenvolvimento, o objeto foi utilizado em duas aulas (com intervalo de sete dias entre elas), permitindo-nos avaliar sua qualidade e realizar considerações sobre o objeto e o método.

O restante deste trabalho está organizado da seguinte forma. A Seção 2 apresenta os principais conceitos relacionados à PBL. Considerando PBL e LODM, na Seção 3 descrevemos, sucintamente, o método para desenvolvimento de OA com PBL considerado neste estudo. As Seção 4 e Seção 5 contém, respectivamente, o relato sobre o desenvolvimento do OA e o uso deste objeto com um grupo de alunos dos cursos de Engenharias. Trabalhos relacionados são apresentados na Seção 6. Finalmente, apresentamos as conclusões e perspectivas para futuros trabalhos na Seção 7.

\section{Aprendizagem Baseada em Problema - PBL}

A Aprendizagem Baseada em Problemas (PBL) é uma abordagem em que os alunos têm o objetivo de resolver um problema, deixando de ser receptores do conhecimento passado pelo professor e se tornando o condutor principal do próprio aprendizado. A ideia principal da PBL é buscar que o próprio aluno construa seu conhecimento e que ele se interesse em resolver o problema antes de receber os conteúdos, mudando sua postura no aprender. Assim o aluno adquire uma autonomia e mostra que pode compreender determinados assuntos ou conhecimentos com sua própria visão. O professor tem um papel diferenciado do modo tradicional de ensinar e se torna um facilitador para que seus alunos cumpram as etapas de resolução do problema apresentado [Gil 2015].

Tipicamente, a abordagem PBL tem início com a apresentação de um problema aos alunos, sem nenhuma informação e instrução sobre a solução do problema. $\mathrm{O}$ problema em si tem a finalidade de fazer o alunos, individualmente ou em grupo, estudar os assuntos relacionados analisando o problema e possíveis soluções. Quando os alunos identificam as questões importantes, eles realizam o estudo individual antes de se reunir com o grupo para que todos os membros troquem informações e 
conhecimentos pesquisados e ainda os da sua experiência e vivência, para tentar resolver o problema [Mamede 2001]. A fase final da abordagem envolve a atividade de reflexão para que os alunos avaliem seu próprio progresso e também o dos outros membros do grupo [Ribeiro 2008].

\section{Método - LODM + PBL}

O LODM (Learning Object Development Method) é um método de desenvolvimento de OA [Graciotto Silva et al. 2011] derivado do IMA-CID [Barbosa e Maldonado 2011], composto de três modelos: conceitual, instrucional e de interação. Os modelos são definidos de forma iterativa, conforme apresentado na Figura 1. Inicia-se pelo modelo conceitual, representando-se os objetivos educacionais por conceitos e proposições em um mapa conceitual [Novak 1977]. A partir deste, cria-se o modelo instrucional, definindo-se itens de informação e atribuindo-lhe um papel instrucional dentro da estrutura da atividade de aprendizagem. Finalmente, o modelo de interação estabelece a sequência de instrução e as interações aplicáveis ao objeto de aprendizagem. A partir do modelo de interação, procede-se à geração do objeto de aprendizagem para a plataforma de execução de sua escolha (apresentação tradicional ou programa para televisão digital).

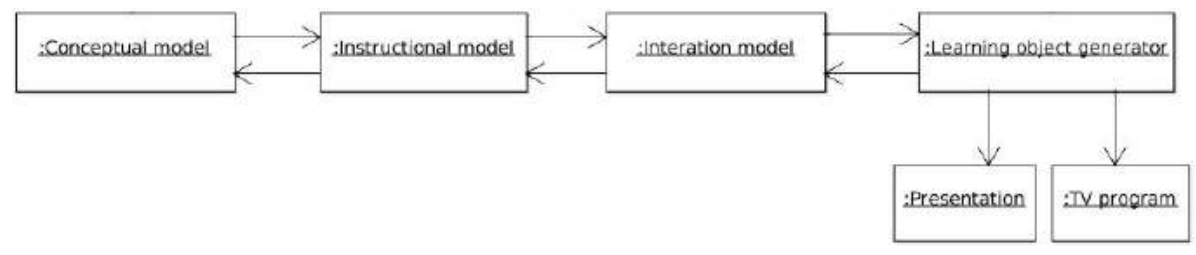

Figura 1. Visão geral dos modelos empregados no LODM [Graciotto Silva 2012].

Considerando as características e requisitos das atividades de aprendizagem com PBL, observa-se que os elementos chaves de PBL são a formulação de questões, que podem ser exploradas e respondidas através de uma investigação sistemática e auto-dirigida, e o teste e a revisão das hipóteses através da aplicação dos conhecimentos recentemente adquiridos. Para o processo são essenciais a discussão ativa e a análise dos problemas, hipóteses, mecanismos e tópicos de aprendizagem, capacitando assim os estudantes a adquirir e aplicar conhecimentos, permitindo-os colocar em prática as habilidades de comunicação e trabalho em equipe.

Para a representação dos elementos necessários ao PBL nos modelos do LODM, mais especificamente ao modelo instrucional, foram definidos uma coleção de elementos do tipo PBL Element com os seguintes esteriótipos: Problem, Solution e Team, conforme mostrado na Figura 2.

O esteriótipo Problem representa um item de informação que apresenta uma situação real. Deve ter características específicas da área de conhecimento como o nome, o enunciado e os conceitos a serem abordados e aprendidos. O esteriótipo Solution representa um item de informação que busca que os membros do grupo (alunos) apresentem a solução ou soluções para o problema, levando em consideração as características do problema, o material fornecido pelo professor, as informações buscadas ou pesquisadas durante a atividade PBL e os conhecimentos adquiridos durante a vida. O esteriótipo Team representa os grupos de alunos formados no início da atividade PBL, 


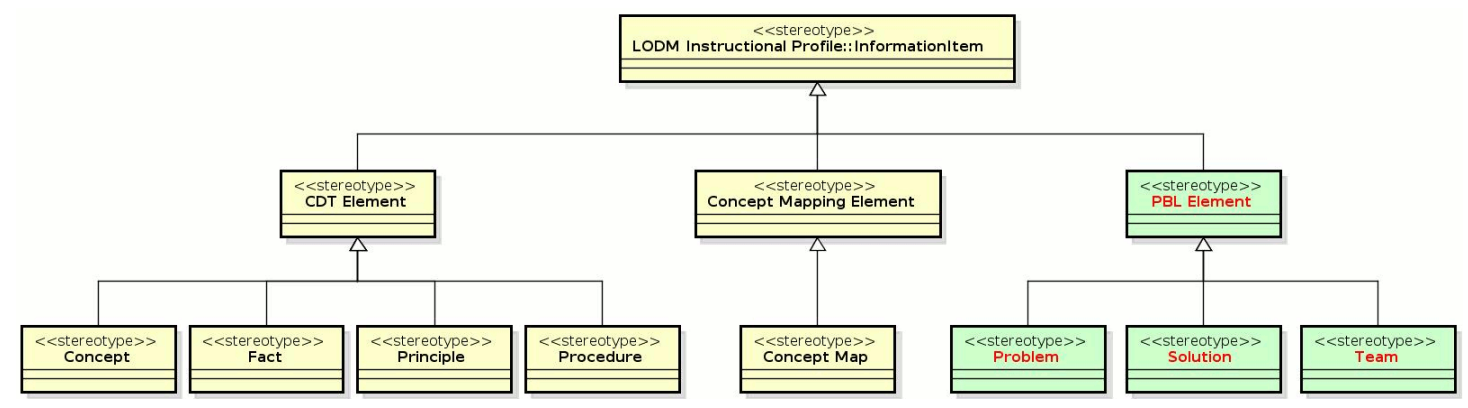

Figura 2. Elementos de PBL adicionados ao LODM.

em que os membros devem eleger um coordenador e um relator. $\mathrm{O}$ coordenador tem a função de organizar o trabalho em equipe. O relator é responsável por fazer as anotações que serão utilizadas para a elaboração dos relatórios.

\section{Desenvolvimento}

O estudo foi conduzindo para o contexto de alunos dos primeiros períodos de cursos de Engenharia e para o ensino de Computação. Para o desenvolvimento do OA foi utilizando o método LODM com PBL, conforme apresentado na Seção 3, organizando-se em três fases: análise, design e desenvolvimento.

Para o início do desenvolvimento do OA foi necessário fazer uma análise para se definir o curso, a área, a disciplina, os objetivos educacionais e o perfil do aluno. Estes objetivos foram definidos, criando-se o modelo conceitual na forma de um modelo de conhecimento estruturado em conceitos e proposições.

Na fase de Design, com o problema definido, fez-se a análise do conteúdo utilizando os mapas criados segundo o LODM, analisando os OA's existentes, as anotações e rascunhos, para se definir a estrutura do OA. Conhecendo os conceitos, definiu-se a organização das informações necessárias para as execuções das atividades de aprendizagem com o modelo instrucional, apresentado na Figura 3. Os elementos do contexto da viga engastada são trabalhados em paralelo às atividades que buscam trabalhar conceitos de programação de computadores. Especificamente quanto aos conceitos de computação, duas unidades de instrução foram definidas, aprofundando-se os conceitos esperados para a solução do problema de viga engastada. Em seguida o modelo de interação complementou o modelo instrucional, definindo elementos instrucionais e reorganizando os elementos do modelo instrucional, definindo assim as atividades de aprendizagem em função da sincronização entre os elementos do modelo.

Concluído o design do OA, seguiu-se à fase de Desenvolvimento, criando-se o material com base nos requisitos estabelecidos no modelo conceitual e no projeto especificado no modelo de interação. Também foram preparados os pacotes para alunos e professor, com esquemas de aula, vídeos, materiais impressos contendo o OA em si ${ }^{1}$.

\footnotetext{
${ }^{1} \mathrm{O}$ objeto de aprendizagem desenvolvido está disponível em https://github.com/ NarciNogueira/OA_Viga-engastada/.
} 


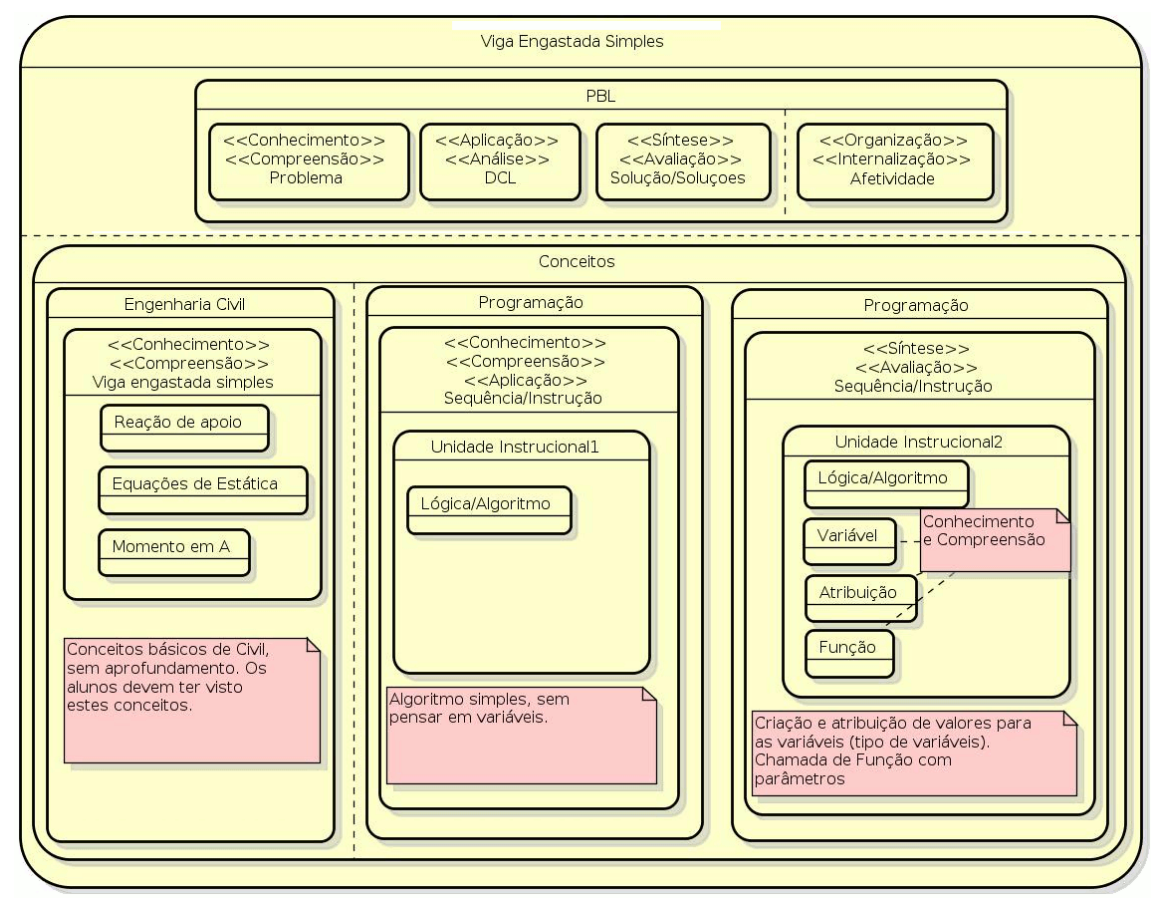

Figura 3. Modelo instrucional do OA sobre viga engastada e programação.

\section{Utilização}

Com o OA criado, procedeu-se à utilização conforme relatado a seguir. A atividade foi planejada para durar dois dias (com intervalo de sete dias entre eles), fora de horário de aula, viabilizando a livre participação dos alunos. Foram convidados alunos dos primeiros períodos dos cursos de Engenharia que cursavam disciplinas sobre Computação (Fundamentos de Programação e Computação I) da UTFPR, totalizando 124 alunos convidados. Após o período de inscrição, nove alunos confirmaram a participação e sete efetivamente participaram das atividades.

Para cada dia de atividade, uma unidade instrucional, parte do objeto de aprendizagem criado, foi entregue aos alunos. Nas próximas duas subseções, cada uma dessas unidades é descrita.

\subsection{Unidade instrucional 1}

Antes da entrega da primeira unidade instrucional, foi realizada uma breve explanação de como seria a atividade. Dois vídeos sobre a metodologia PBL foram exibidos nos primeiros 10 minutos. Em seguida, os alunos foram distribuídos em dois grupos, sendo um grupo com 4 e outro com 3 alunos (os alunos elegeram o Coordenador e o Relator). Como o problema de viga engastada é tipicamente abordado em Engenharia Civil, cuidado foi tomado para que pelo menos 1 alunos de Engenharia Civil estivesse em cada grupo.

Iniciou-se então a atividade sobre a viga engastada, entregando-se a primeira unidade instrucional, que apresentava o problema e relacionava conceitos elementares de programação, conforme ilustrado na Figura 4. A atividade teve duração de 1h10m. Alguns questionamentos dos grupos foram feitos durante a atividade e todos foram respondidos no momento planejado no esquema de aula, conforme segue na Tabela 1. 


\section{OBJETO DE APRENDIZAGEM (OA) \\ Engenharia Civil (Viga engastada com carga concentrada).}

\section{PACOTE PARA O ALUNO.}

Unidade Instrucional 1: Objeto de Aprendizagem (OA) com elementos de PBL(Problem Based Learning) para o curso de Engenharia Civil, incluindo elementos de PBL no LODM (Learning Object Development Method).

Tema

Problema 1: Viga engastada com carga concentrada.

Desenvolvimento de um diagrama de corpo livre (DCL) e um algoritmo simples com os passos para a solução do Problema.

\section{Problema}

Viga engastada simples com dois pontos, sendo que o primeiro é o apoio (A) e o segundo é onde esta concentrada a carga (B). A carga de $-8 \mathrm{~N}$ esta concentrada no ponto B (livre = é uma viga em balanço) à 2 metros do ponto $\mathrm{A}$ (fixo = encaixada). No ponto $\mathrm{A}$ o apoio é de terceiro gênero (restringe 3 movimentos = na horizontal, na vertical e rotação).

OBS. o valor da carga é negativo devido o sentido (para baixo).

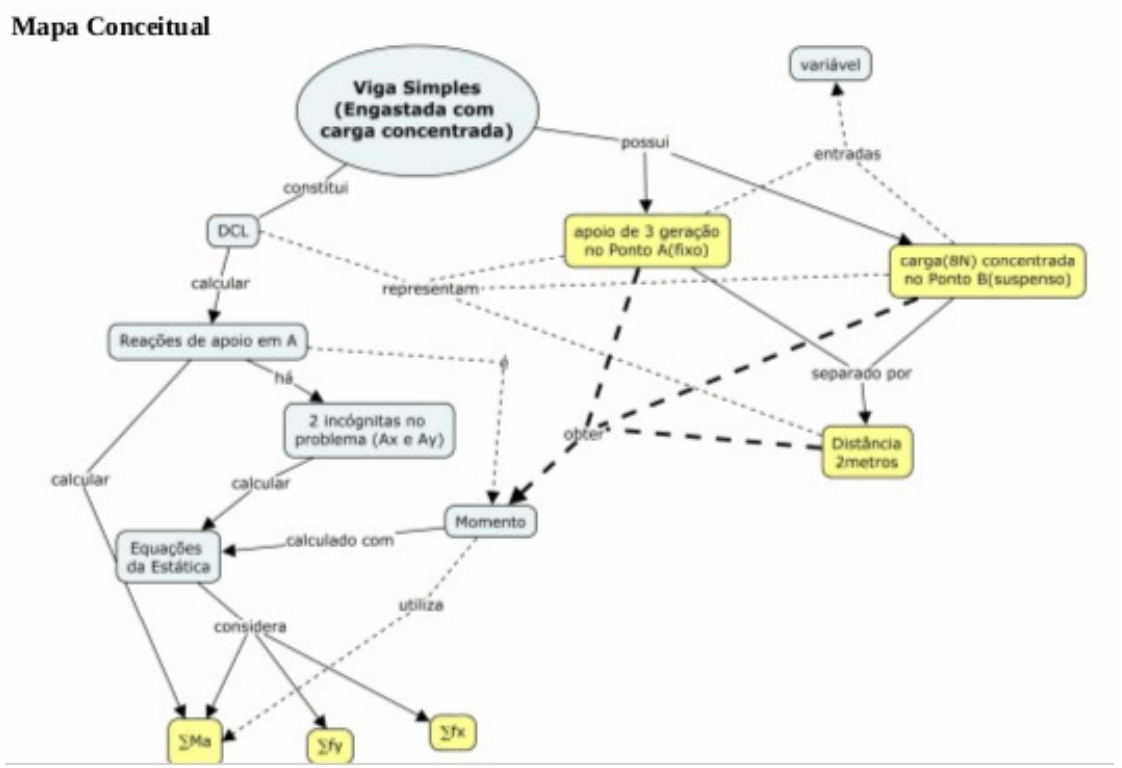

Figura 4. Unidade instrucional utilizada na primeira aula.

Os grupos enviaram o relatório parcial da primeira unidade instrucional no dia seguinte à aula, anexando também o diagrama de corpo livre (DCL) e o algoritmo desenvolvido pela equipe e que soluciona o problema. Os alunos consideram a atividade parcial como estimuladora e os conceitos iniciais de programação foram mostrados de maneira diferente e mais leves do que a forma tradicional de aula em laboratório.

\subsection{Unidade instrucional 2}

Após uma semana, foi realizada nova atividade, entregando-se a segunda unidade instrucional. Os detalhes do PBL foram relembrados nos primeiros 10 minutos e os vídeos utilizados na unidade instrucional 1 foram reprisados. O produto final esperado foi uma função em Python resolvendo o problema, conforme apresentado na Figura 5.

Finalizando a unidade instrucional 2, foi recebido o relatório final de cada grupo, acrescido do algoritmo contendo os conceitos de programação de variáveis e atribuição 
Tabela 1. Segmento da Aula 1

\begin{tabular}{|c|c|c|c|}
\hline $\begin{array}{l}\text { Segmento } \\
\text { de aula }\end{array}$ & Tempo & $\begin{array}{l}\text { Dinâmica } \\
\text { da aula }\end{array}$ & Atividade \\
\hline \multirow{2}{*}{1} & \multirow{2}{*}{$10 \mathrm{~min}$} & \multirow{2}{*}{$\begin{array}{l}\text { Conduzida } \\
\text { pelo professor }\end{array}$} & Explanação sobre PBL e sobre o Problema (vídeo, etc) \\
\hline & & & Divisão das equipes \\
\hline \multirow{4}{*}{2} & \multirow{4}{*}{$40 \mathrm{~min}$} & \multirow{4}{*}{$\begin{array}{l}\text { Conduzida } \\
\text { pelos alunos }\end{array}$} & Definição dos papeis (Coordenador/Lider, Relator) \\
\hline & & & $\begin{array}{l}\text { Conversa sobre a visão de cada membro e } \\
\text { divisão das tarefas }\end{array}$ \\
\hline & & & Resolução do Problema, \\
\hline & & & Elaboração do relatório parcial \\
\hline \multirow{2}{*}{3} & \multirow{2}{*}{$10 \mathrm{~min}$} & \multirow{2}{*}{$\begin{array}{l}\text { Conduzida } \\
\text { pelo professor }\end{array}$} & Debate entre professor e alunos \\
\hline & & & Síntese do professor \\
\hline 4 & $5 \mathrm{~min}$ & $\begin{array}{l}\text { Conduzida } \\
\text { pelos alunos }\end{array}$ & $\begin{array}{l}\text { Entrega do relatório parcial ou definição de } \\
\text { data para enviar o produto da atividade. }\end{array}$ \\
\hline
\end{tabular}

de valores à estas variáveis, utilizando o valores correspondentes ao problema e Código Python com a função resolvendo o problema.

\subsection{Avaliação dos resultados}

Avaliamos a qualidade dos OA considerando a visão dos alunos. Abaixo algumas relatos dos alunos que reforçam que o modelo e a metodologia PBL colaboraram para o estímulo e melhora no entendimento dos objetivos educacionais propostos.

- "Essa atividade mostrou o quão despreparados nós estamos em fazer algo relativamente sozinho, sem ajuda de um tutor, isso mostra que deveria ter mais incentivo desde a pré escola, para que no futuro nós conseguimos realizar esse tipo de atividade no dia a dia".

- "Devido ao fim de semestre, acabei não dando muito enfoque ao curso. Todavia, o problema relacionado e a metodologia utilizada me influenciaram a comparecer nas atividades presenciais e auxiliar os demais membros na busca por resultados eficientes. Acredito que poderia ter dado mais de mim".

- "Levando em consideração o meu nível de conhecimento, o método proposto auxiliaria bastante para motivar a aprender mais."

Desta forma, observa-se que os alunos demonstraram interesse e estavam motivados em realizar a atividade. Corrigidos os relatórios, observou-se também que os objetivos de aprendizagem foram alcançados pelos grupos.

\section{Trabalhos Relacionados}

A aplicabilidade da PBL como método de ensino de computação para cursos de Engenharia é feita através da implementação de estudos relacionados a problemas específicos de cada área da engenharia [Mahadevan-Jansen et al. 2003, Sun et al. 2012, Zachary 1996].

Por exemplo, um dos primeiros trabalhos sobre PBL em Computação estabelece que o método de resolução de problemas computacionais consiste em 5 etapas: entender o problema e saber exatamente o que fazer para resolver; criar um modelo matemático; criar o método de resolução do modelo matemático; aplicar o método na linguagem de programação C; e avaliar a solução [Zachary 1996]. 


\title{
OBJETO DE APRENDIZAGEM (OA) \\ Engenharia Civil (Viga engastada com carga concentrada).
}

PACOTE PARA O ALUNO.

Unidade Instrucional 2: Objeto de Aprendizagem (OA) com elementos de PBL(Problem Based Learning) para o curso de Engenharia Civil, incluindo elementos de PBL no LODM (Learning Object Development Method).

Problema 1 : Viga engastada com carga concentrada.

Tema

Acrescentar ao algoritmo os conceitos de programação de variáveis e atribuição de valores a estas variáveis utilizando o valores correspondentes ao problema e desenvolver uma função em Python resolvendo o problema.

\begin{abstract}
Problema
Viga engastada simples com dois pontos, sendo que o primeiro é o apoio (A) e o segundo é onde esta concentrada a carga (B). A carga de $-8 \mathrm{~N}$ esta concentrada no ponto B (livre = é uma viga em balanço) à 2 metros do ponto $\mathrm{A}$ (fixo = encaixada). No ponto $\mathrm{A}$ o apoio é de terceiro gênero (restringe 3 movimentos = na horizontal, na vertical e rotação). OBS. o valor da carga é negativo devido o sentido (para baixo).
\end{abstract}

\section{CONCEITOS :}

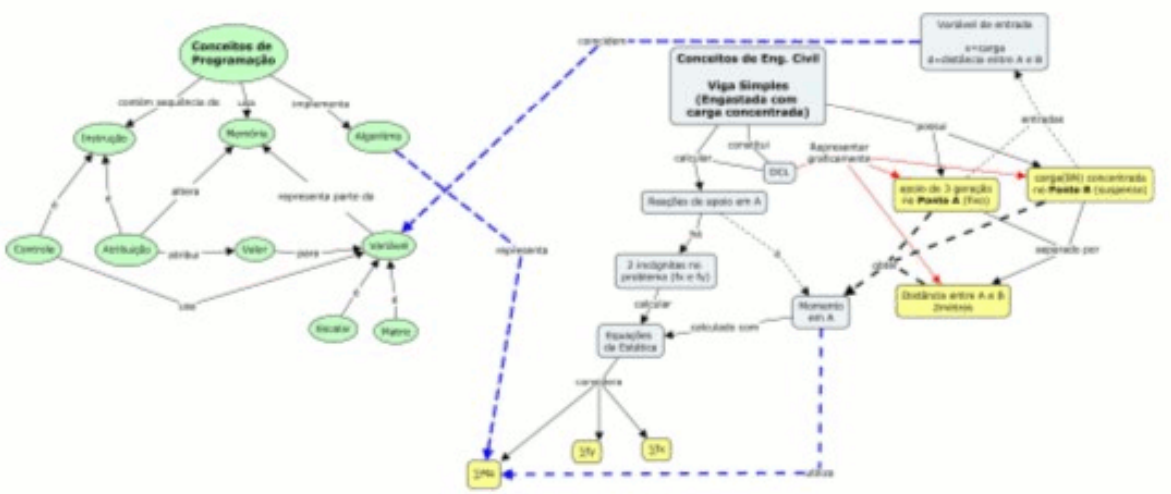

Figura 5. Unidade instrucional utilizada na segunda aula.

Em outro artigo, [Sun et al. 2012] relatam a experiência de um curso de introdução a computação para cursos de engenharia, com a disponibilização dos vídeos dos slides das palestras (material online), tentando suprir a falta de aulas teóricas. Após os alunos assistirem os vídeos eles vão ao laboratório fazer os exercícios práticos baseados na resolução de problemas (PBL) com a supervisão de instrutores.

Ainda, [Silva e Borges 2016] apresentam uma proposta de ensino de conceitos de lógica de programação utilizando kit robótico como ferramenta pedagógica baseada no método Problem-Based Learning (PBL) e na Aprendizagem Colaborativa. Este proposta visa demonstrar maneiras para trabalhar alguns conceitos da LP com objetivo de identificar o melhor uso dos recursos oferecidos pelo kit, buscando despertar o interesse dos alunos propiciando a melhor compreensão dos conceitos trabalhados.

Também [Brauner et al. 2016] apresentam um evento de ensino que estimula a prática multidisciplinar do ensino de computação através de uma competição ou maratona 
(Hackathons). Este evento de programação foca a resolução de problemas através de uma dinâmica colaborativa e competitiva. As atividades desta maratona foram realizadas eletronicamente pela página Web http://computacao.cc/codearena/. Estas atividades foram: chamada de ideias; formação das equipes(times); reunião de aquecimento; apresentações dos produtos a etapa de premiação.

Por fim, [Araújo et al. 2015] relatam a experiência, com ênfase na prática docente, de um minicurso de lógica de programação planejado e executado conforme os princípios da metodologia PBL, com o objetivo de estimular o pensamento computacional em crianças do ensino fundamental. As principais ações associadas ao planejamento do minicurso, referem-se à definição de objetivos educacionais claros e precisos conforme a Taxonomia de Bloom Revisada [Anderson e Krathwohl 2001].

\section{Conclusões}

Apresentamos neste artigo a aplicação do método de desenvolvimento LODM com elementos de PBL na criação de OA para ensino de Computação para cursos de Engenharia. Além disso, foi realizado um experimento usando o OA criado em turmas de primeiros e segundos períodos dos cursos de Engenharia no Câmpus Campo Mourão da UTFPR. Foram aplicadas duas unidades instrucionais, seguindo etapas planejadas, em que os produtos finais, relatórios parciais e finais e respostas do questionários provêm indícios de que o modelo aperfeiçoado ao LODM e a metodologia PBL colaboram para o estímulo e melhora no entendimento dos objetivos educacionais propostos.

Analisando-se o desenvolvimento do objeto de aprendizagem, observou-se que o método auxilou satisfatoriamente no entendimento das características e requisitos de PBL para criação e aplicação de objetos de aprendizagem. A criação de elementos no LODM para representar PBL foi uma das atividades importantes para o entendimento do método e para a criação de OA's completos e robustos, que atendam os fins educacionais das disciplinas de Computação e consequentemente do curso como um todo.

Como trabalhos futuros, planejamos desenvolver outros OA com PBL com o método utilizado neste trabalho, analisando tanto a qualidade do objeto gerado quanto o esforço necessário para seu desenvolvimento. Estes novos estudos permitirão a comparação dos resultados com técnicas tradicionais de desenvolvimento de OA, viabilizando a avaliação da eficácia e da eficiência do método e dos OA gerados.

\section{Referências}

Albright, P. (2005). Open educational resources final forum report. Technical report, UNESCO, Paris, França. Internet Discussion Forum: Open Educational Resources Open Content for Higher Education.

Anderson, L. W. e Krathwohl, D. R., editors (2001). A Taxonomy for Learning, Teaching, and Assessing: A Revision of Bloom's Taxonomy of Educational Objectives. Longman, New York, NY, EUA.

Araújo, D. d. C. a., Rodrigues, A. N., de Araújo Silva, C. V., e Soares, L. S. (2015). O ensino de computação na educação básica apoiado por problemas: Práticas de licenciandos em computação. In: XXXV Congresso da Sociedade Brasileira de Computação - XXIII WEI - Workshop sobre Educação em Computação, Recife, PE, Brasil. SBC. 
Barbosa, E. F. e Maldonado, J. C. (2011). IMA-CID: an integrated modeling approach for developing educational modules. Journal of the Brazilian Computer Society, 17(4):207-239.

Bosse, Y. e Gerosa, M. A. (2015). As disciplinas de introdução à programação na USP: um estudo preliminar. In: XXXV Congresso da Sociedade Brasileira de Computação - XXIII WEI - Workshop sobre Educação em Computação, p. 1389-1397, Recife, PE, Brasil. SBC.

Brauner, D., Margreff, P., Tavares, T., da Costa, V. K., e Silva, A. L. (2016). Estímulo à prática multidisciplinar no ensino de computação e design através de um evento de programação focado em problemas. In: XXXVI Congresso da Sociedade Brasileira de Computação - XXIV WEI - Workshop sobre Educação em Computação, p. 2126-2135, Porto Alegre, RS, Brasil. SBC.

Gil, A. C. (2015). Didática do Ensino Superior. Atlas, São Paulo, SP, Brasil.

Graciotto Silva, M. A. (2012). LOD: uma abordagem para desenvolvimento de objetos de aprendizagem multimídias e interativos. Tese de Doutorado, Universidade de São Paulo, São Carlos, SP, Brasil.

Graciotto Silva, M. A., Barbosa, E. F., e Maldonado, J. C. (2011). Model-driven development of learning objects. In: 41st ASEE/IEEE Frontiers in Education Conference, p. F4E-1-F4E-6, Rapid City, South Dakota, EUA. IEEE.

Mahadevan-Jansen, A., Rowe, C. J., Crocetti, J., e Brophy, S. (2003). A paradigm shift in the approach to freshman engineering education. In: ASEE 2003 Annual Conference, p. 11570-11585, Nashville, Tennessee, EUA. ASEE.

Mamede, S. (2001). Aprendizagem baseada em problemas: Características, processos e racionalidade. In: Aprendizagem baseada em problemas: anatomia de uma abordagem educacional, cap. 2, p. 27-48. Hucitec, Fortaleza, CE, Brasil.

Novak, J. D. (1977). A theory of education. Cornell University Press, Ithaca, NY,EUA.

Ribeiro, L. R. d. C. (2008). Aprendizagem baseada em problemas (PBL): uma experiência no ensino superior. EdUFSCar, São Carlos, SP, Brasil, 1 ed.

Silva, F. Z. N. e Borges, M. A. F. (2016). PBL e robótica no ensino de conceitos de lógica de programação. In: XXXVI Congresso da Sociedade Brasileira de Computação XXIV WEI - Workshop sobre Educação em Computação, Porto Alegre, RS, Brasil. SBC.

Sun, L., Kindy, M., Liron, C., Grant, C. D., e Waterhouse, S. A. (2012). Hybrid course design: Leading a new direction in learning programming languages. In: 119th ASEE Annual Conference and Exposition, p. 1-13, San Antonio, TX, EUA. ASEE.

Wiley, D. A. (2000). Learning Object Design and Sequencing Theory. Tese de Doutorado, Department of Instructional Psychology and Technology - Brigham Young University, Provo, Utah, EUA.

Zachary, J. L. (1996). Introduction to computing for engineers: New approaches to content and pedagogy. In: 26th Annual Conference on Frontiers in Education, p. 149-153, Piscataway, NJ, EUA. IEEE. 